\title{
Efficient readout of micromechanical resonator arrays in ambient conditions
}

\author{
W. J. Venstra ${ }^{\text {a) }}$ and H. S. J. van der Zant ${ }^{\text {b) }}$ \\ Kavli Institute of Nanoscience, Delft University of Technology, Lorentzweg 1, 2628CJ Delft, \\ The Netherlands
}

(Received 23 October 2008; accepted 15 November 2008; published online 11 December 2008)

\begin{abstract}
We present a method for efficient spectral readout of mechanical resonator arrays in dissipative environments. Magnetomotive drive and detection are used to drive double clamped resonators in the nonlinear regime. Resonators with almost identical resonance frequencies can be tracked individually by sweeping the drive power. Measurements are performed at room temperature and atmospheric pressure. These conditions enable application in high throughput resonant sensor arrays. (C) 2008 American Institute of Physics. [DOI: 10.1063/1.3042097]
\end{abstract}

Micrometer and nanometer scale mechanical resonators are widely considered as mass and force sensors. Adsorption or desorption of molecules by the resonator are detected as slight changes in resonance frequency. Several methods exist to measure the resonance frequency of a single resonator. ${ }^{1-4}$ Small arrays of static mechanical sensors be scanned optically ${ }^{5}$ or the reflection of the array as a whole may be analyzed. ${ }^{6}$ Spectral readout of small-scale resonant arrays has been reported in vacuum. ${ }^{7}$ In this letter, we present an efficient method to readout resonators with closely spaced resonance frequencies in dissipative environments. We show that the number of resonant sensors, which can be operated in a given bandwidth, is not limited by the $Q$ factor. Using a magnetomotive setup, we can discriminate mechanical resonators with almost identical frequencies in ambient conditions.

To track two individual resonators with quality factor $Q$ in a frequency spectrum, their resonance frequencies $f_{i}$ should be separated by at least $\Delta f=f_{i} / Q$. Simultaneous operation of $n$ resonators requires a minimum bandwidth on the order of $\Delta f=f_{1}(1+1 / Q)^{n}$. Here $f_{1}$ is the resonance frequency of the slowest resonator. In vacuum, the $Q$ factor of a mechanical resonator can be of the order $10^{4}$ (Ref. 7) and readout of large arrays of resonators should be feasible. At atmospheric pressure, however, $Q$ factors usually drop below $10^{2}$ because of viscous drag. ${ }^{8}$ This limits the number of resonators within a practical bandwidth to a few hundred at most. Large-scale arrays therefore require either multiple detectors or separated drive circuits, and the complexity of such systems rapidly increases with the number of resonators.

To address this problem, we propose to readout arrays of resonators with closely spaced center frequencies by sweeping the drive power from low to high values at constant frequency. Once the nonlinear regime is accessed, individual resonators are marked by instantaneous transitions in the phase and amplitude response. When each nonlinear resonator is used as a sensing element, ${ }^{9}$ the locations of these characteristic amplitudes are affected by adsorption or desorption induced mass or stress change to the concerning resonator. We conduct experiments on arrays of double clamped resonators at room temperature and atmospheric

\footnotetext{
a) Electronic mail: w.j.venstra@tudelft.nl.

${ }^{b)}$ Electronic mail: h.s.j.vanderzant@tudelft.nl.
}

pressure. We experimentally show that in a two-dimensional (2D) array of six resonators with closely spaced resonance frequencies, individual resonators can be discriminated using a single drive and detector unit. Calculations confirm the experimental findings.

Arrays of double clamped mechanical resonators are fabricated out of $100 \mathrm{~nm}$ thick low-pressure chemical vapor deposited (LPCVD) silicon nitride by electron beam lithography. All resonators have the same dimensions 200 $\times 15 \mu \mathrm{m}^{2}$. Slight variations in center frequencies of the resonators are expected as a result of variations in residual stress. ${ }^{10}$ Figure 1(a) shows one of the resonators before metallization. A layer of chromium and gold is evaporated on top to enable magnetomotive drive and detection. The resonators share a support on one side, while supports at the other side are separated. This allows the measurement of both the individual and the collective responses.

Figure 1(b) shows the measurement setup schematically. The rf voltage from a network analyzer is converted to a current, which drives the resonators (two in series depicted). A magnetic field of $1.9 \mathrm{~T}$ is generated by a Halbach ${ }^{11}$ array constructed from NdFeB permanent magnets. The resulting voltage is amplified and measured by the network analyzer. This measurement setup can be used at room temperature and atmospheric pressure, in contrast to earlier magnetomotive experiments in vacuum and/or at cryogenic temperatures. As a result of viscous drag, the $Q$ factors in our experiments are a factor of 10 to 100 lower than in those experiments.

Figure 2(a) shows the linear response of two resonators with almost identical center frequencies. The voltage drop due to the resistance of the resonator, bond wires, etc., has been subtracted. The resonance frequencies and $Q$ factors
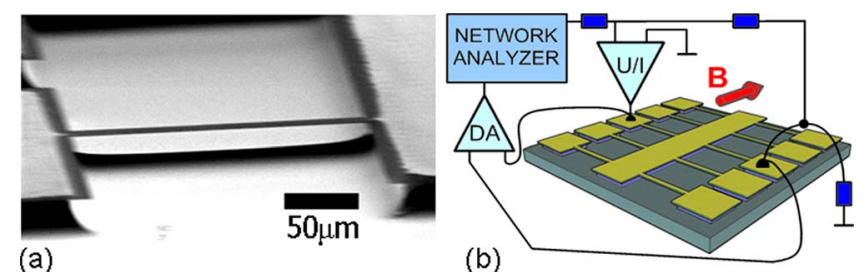

(b)

FIG. 1. (Color online) (a) Scanning electron micrograph of a single resonator. (b) Magnetomotive measurement setup. The current source is formed by the amplifier U/I and the resistor network. 


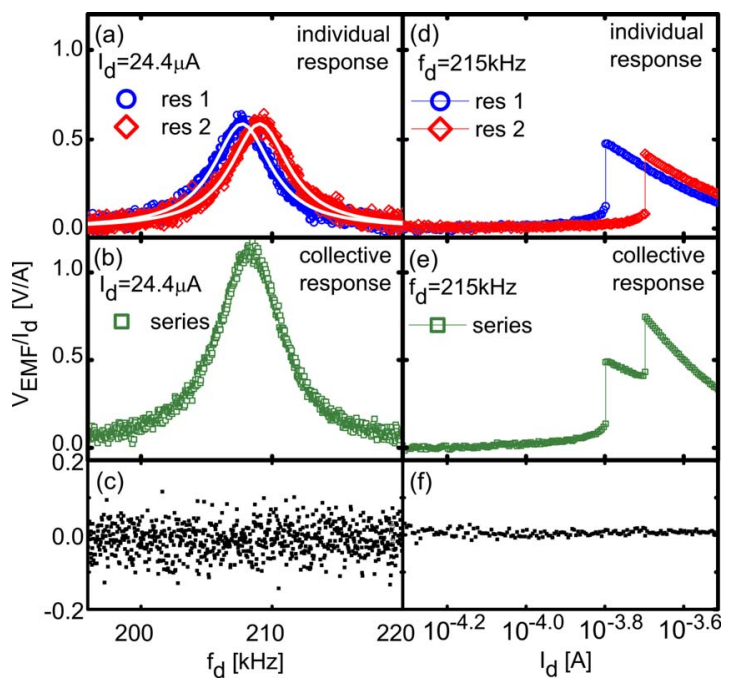

FIG. 2. (Color online) Magnetomotive measurements in ambient environment. (a) Individual linear frequency response of two resonators with almost identical resonance frequencies. (b) The same resonators driven nonlinear by sweeping drive power at $215 \mathrm{kHz}$. [(c) and (d)] Collective response of the resonators connected in series in linear (c) and nonlinear (d) regimes. [(e) and (f)] show the difference between the calculated sum of the individual responses and the measured collective response in the linear (e) and the nonlinear (f) regimes.

are determined by fitting Lorentzian functions (shown in the figure) to the measured data. The resonance frequencies of these devices were 207.72 and $209.05 \mathrm{kHz}$, and the $Q$ factor is 39 for both resonators. The corresponding bandwidth is $5.4 \mathrm{kHz}$, whereas the difference in resonance frequency is $1.3 \mathrm{kHz}$. Now the resonators are connected in series and the collective response is measured. Figure 2(b) shows the result for the same drive conditions as in (a). Discrimination between the two resonators is impossible as the resonator bandwidth is more than four times the difference in center frequency. We now individually drive the same resonators at increasing power at a constant frequency of $215 \mathrm{kHz}$. Figure 2(d) shows the result: a steep transition in the resonator amplitude marks the characteristic drive amplitude at this frequency. When the collective response is measured at strong driving, as in (d), the two resonators are easily distinguished.

In the absence of interaction, the response of multiple resonators is just the sum of the individual responses. To verify this, the difference between the sum of the individual responses of (a) and (d) and the collective response in (b) and (e) is plotted in Figs. 2(c) and 2(f). No amplitude dependency is found, which indicates that the coupling is weak. Note that a high driving power results in a notably lower noise level in the nonlinear driven system. ${ }^{12}$

We now turn to a $2 \mathrm{D}$ array of six resonators, for which the arrangement and the measured linear resonance frequencies are depicted in the inset of Fig. 3(a). In this experiment, the bandwidth of the individual resonators was $5 \pm 0.1 \mathrm{kHz}$, which corresponds to a $Q$ factor of approximately 50 .

Figure 3(a) shows the nonlinear amplitude response at $f_{d}=270 \mathrm{kHz}$; clearly six resonators can be distinguished. In Figs. 3(b) and 3(c), sweeps at fixed drive frequencies ranging from 250 to $285 \mathrm{kHz}$ are plotted. Six lines marking characteristic jumps in resonator amplitude and phase remain equidistant. Indeed, over the nonlinear regime, the total bandwidth covering the six resonators changes by less than $60 \mathrm{~Hz}$ in this measurement. When sweeping frequency from low to
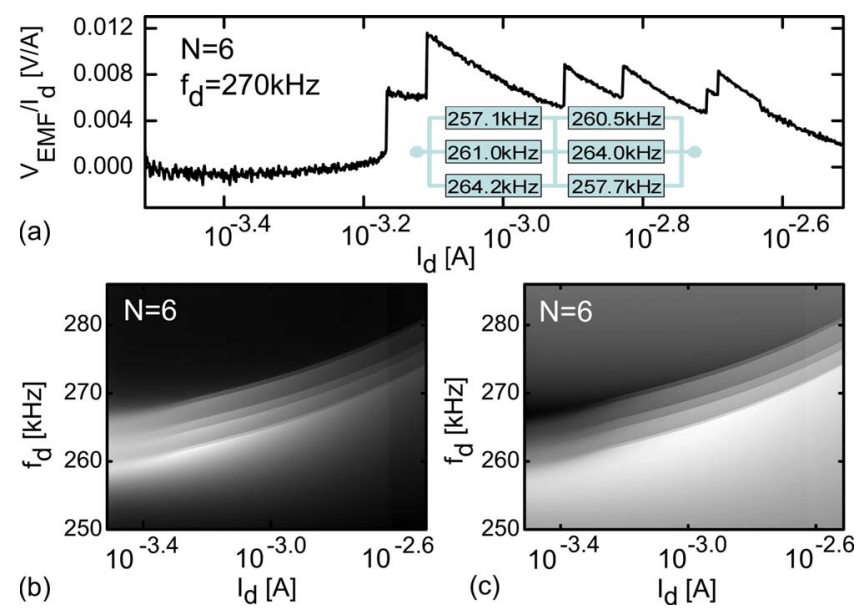

FIG. 3. (Color online) Measurements on a 2D array of six resonators. (a) Resonator response while sweeping the drive current at $f=270 \mathrm{kHz}$. The inset shows the arrangement of the resonators and their linear resonance frequencies. (b) and (c) show amplitude and phase response of the array driven at different frequencies $f_{d}$.

high values at constant drive current (not shown), we found strong coupling between the resonators, which causes multiple resonators to collapse simultaneously at high driving power. Strong coupling makes the system useless as an array of independent sensors.

As for the differences in the center frequencies of the resonators, we note that in LPCVD silicon nitride residual stress variations in the order of $10 \%$ across a $2^{\prime}$ wafer are not unusual. ${ }^{10}$ Using this number, the residual stress gradient would be on the order of $100 \mathrm{~Pa} / \mu \mathrm{m}$. Given the distance between the resonators (at least $300 \mu \mathrm{m}$ ) the difference in residual stress is on the order of $10^{4} \mathrm{~Pa}$, which results in frequency differences on the order of $100 \mathrm{~Hz}$. Similar frequency differences can be obtained by slightly varying the geometry of the resonators or the thickness of the deposited gold layer.

To corroborate the experimental results, we have calculated the characteristic driving amplitude of a nonlinear resonator at a given driving frequency by solving the equation of motion

$$
M \ddot{y}+C \dot{y}+\left(K_{t}+K_{b}\right) y+K_{3} y^{3}=B L I_{d} \cos (\omega t),
$$

where $M$ is the resonator effective mass, $y$ is the beam displacement, $C$ is the damping constant, and $K_{t}$ and $K_{b}$ represent linear stiffness terms due to residual tension and bending rigidity, respectively. The nonlinear term $K_{3}$ is a result of the elongation of the beam, $L$ is the beam length, $B$ is the magnetic field strength, and $I_{d}$ is the driving current.

For the beams in Fig. 2, the average residual tension $T_{0}=74 \mu \mathrm{N}$ is found by comparing the calculated stress-free resonance frequency to the measured resonance frequency. ${ }^{13}$ We calculated that $K_{t} / K_{b} \approx 85$, which indicates that the bending rigidity of the resonators is insignificant and the linear resonance frequency is merely determined by $M$ and $K_{t}$. The magnetomotive voltage generated by the stringlike linear resonator at resonance equals 

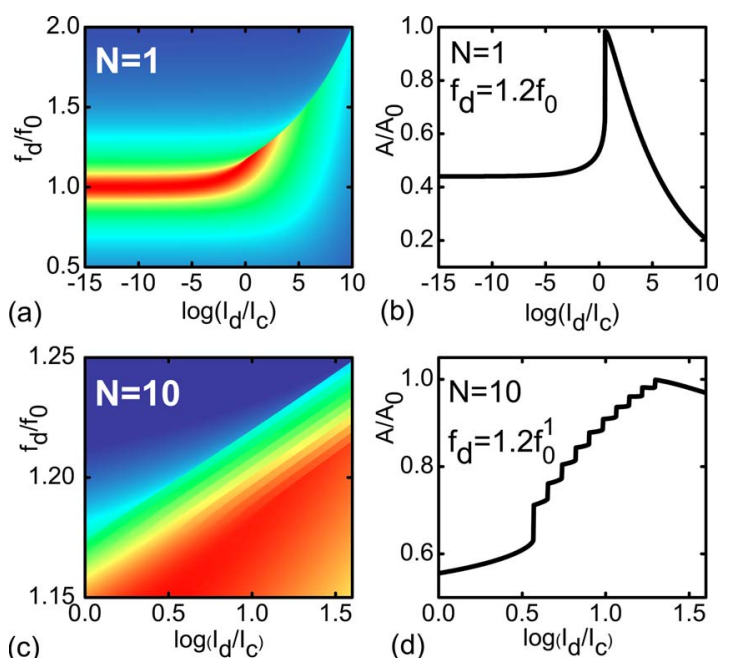

FIG. 4. (Color online) Calculated amplitude responses. (a) Normalized amplitude response of a single resonator driven at fixed frequency as a function of the normalized drive amplitude $f_{d} / f_{0}$. (b) Cross section at $f_{d} / f_{0}=1.2$. (c) and (d) show the amplitude response for ten resonators.

$$
V_{E M F}=\frac{4}{\pi^{4}} \frac{\omega_{1} I_{d} B^{2} Q L^{3}}{T_{0}} .
$$

With the experimental values $B=1.9 \mathrm{~T}, Q=39$, and $L$ $=200 \mu \mathrm{m}$, the transduction at resonance equals $V_{E M F} / I_{d}$ $=0.8 \mathrm{~V} / \mathrm{A}$.

Instead of the frequency-dependent resonator amplitude, we now calculate the resonator amplitude while varying the drive amplitude at constant frequency $f_{d}$ to mimic the experimental situation. Thus, out of two stable states, the resonator always vibrates at the lowest amplitude. Figure 4(a) shows the calculated response for a resonator similar to the ones used in the experiments. The frequency axis is normalized to the linear resonance frequency $f_{0}$, and traces for the normalized drive frequencies $f_{d} / f_{0}$ from 0.5 to 2.0 are combined in (a). Red regions correspond to a high amplitude. The trace for $f_{d} / f_{0}=1.2$ is shown in Fig. 4(b). Sweeping parallel to the horizontal axis, locations with multivalued resonator amplitudes are accessible at large drive amplitudes, when $f_{d}>f_{0}$. Figure $4(\mathrm{c})$ shows the simulated response of an array of ten resonators described by Eq. (1), where a slight difference in $K_{t}$ results in different characteristic amplitudes for each resonator. We assume a difference in linear resonance frequency for adjacent resonators equal to one-tenth of the resonator bandwidth. Panel (d) shows a cross section of the amplitude response at $f_{d} / f_{0}^{1}=1.2$, where $f_{0}^{1}$ is the linear resonance frequency of the slowest resonator. The phase response (not shown) also displays steep transitions similar to the amplitude, whose location can be determined with high accuracy. ${ }^{14}$ The calculations thus reproduce the experimental findings in detail.

In conclusion, we have demonstrated an efficient way to operate and readout mechanical resonator arrays in dissipative environments. The center frequencies of the linear resonators can be spaced very closely as each resonator is marked by its characteristic drive amplitude at a fixed frequency instead of the linear resonance frequency. As the exponential relation between required bandwidth and the number of resonators is circumvented, this technique may enable the construction of high-throughput resonant sensor arrays.

The authors acknowledge financial support from Koninklijke Philips NV (Grant No. RWC-061-JR-05028) and from the Dutch organizations FOM and NWO (VICI).

${ }^{1}$ D. Karabacak, T. Kouh, C. C. Huang, and K. L. Ekinci, Appl. Phys. Lett. 88, 193122 (2006).

${ }^{2}$ M. Li, H. X. Tang, and M. L. Roukes, Nat. Nanotechnol. 2, 114 (2007).

${ }^{3}$ S. Dohn, O. Hansen, and A. Boisen, Appl. Phys. Lett. 88, 264104 (2006).

${ }^{4}$ H. W. Ch. Postma, I. Kozinsky, A. Husain, and M. L. Roukes, Appl. Phys. Lett. 86, 223105 (2005).

${ }^{5}$ J. Mertens, M. Álvarez, and J. Tamayo, Appl. Phys. Lett. 87, 234102 (2005).

${ }^{6}$ M. Yue, J. C. Stachowiak, H. Lin, R. Datar, R. Cote, and A. Majumdar, Nano Lett. 8, 520 (2008).

${ }^{7}$ P. A. Truitt, J. B. Hertzberg, C. C. Huang, K. L. Ekinci, and K. C. Schwab, Nano Lett. 7, 120 (2007).

${ }^{8}$ C. A. Van Eysden and J. E. Sader, J. Appl. Phys. 101, 114916 (2006).

${ }^{9}$ D. S. Greywall, Meas. Sci. Technol. 16, 2473 (2005).

${ }^{10}$ J. G. E. Gardeniers, H. A. C. Tilmans, and C. C. G. Visser, J. Vac. Sci. Technol. A 14, 2879 (1996).

${ }^{11}$ K. Halbach, IEEE Trans. Nucl. Sci. 30, 3323 (1983).

${ }^{12}$ A. N. Cleland and M. L. Roukes, J. Appl. Phys. 92, 2758 (2002).

${ }^{13}$ A. Bokaian, J. Sound Vib. 142, 481 (1990).

${ }^{14}$ D. S. Greywall, B. Yurke, P. A. Busch, A. N. Pargellis, and R. L. Willet, Phys. Rev. Lett. 72, 2992 (1994). 